\title{
On the Factors and Suggestions Affecting the Level of Food Work
}

\author{
Hailing Wang ${ }^{1}$, Dong $\mathbf{L i}^{{ }^{2 *}}$ \\ ${ }^{1}$ Qingdao Food Inspection Institute, Qingdao 266000, China. \\ ${ }^{2}$ Qingdao Product Quality Inspection Technology Research Institute, Qingdao 266000, China. E-mail: \\ 13853277127@163.com
}

\begin{abstract}
In recent years, with the continuous improvement and optimization of the social and economic level, the quality of life of the people has been significantly improved. Based on this, the public's attention to my country's food work has been reasonably developed, which has effectively promoted the development of food safety work of my country. Through a large amount of data analysis, the researchers pointed out that at this stage, there are still certain shortcomings in the development of food work in my country that need to be improved. In response to this problem, relevant staff have carried out systematic analysis and exploration, aiming to effectively summarize and improve the relevant factors that affect the level of food work in my country, so as to achieve the improvement and optimization of the comprehensive quality of food hygiene and safety management in my country. This article analyzes and summarizes the current food work situation in my country, and puts forward corresponding optimization suggestions at the same time, aiming to effectively promote the improvement of the overall quality of food work in my country, so as to lay the foundation for a reasonable guarantee of food safety.
\end{abstract}

Keywords: Food Work; Work Status; Influencing Factors; Optimization Suggestions; Coping Strategies

A large amount of research data shows that in the process of food safety work, due to the influence of many factors, the power and responsibilities of relevant management departments are often difficult to fully utilize, which is not conducive to the improvement of the overall level of my country's food work. In recent years, in response to this problem, a large number of researchers have conducted in-depth analysis and exploration, aiming to effectively realize the analysis and discussion of the factors affecting the level of food work, so as to reasonably promote the adjustment of related work methods and improve the quality of food work in my country. Optimize and lay the foundation, so as to provide help for the people to rest assured the construction of the table.

\section{The main factors affecting the level of food work in my country}

\subsection{The comprehensive ability of the food management team is relatively weak}

For now, the overall strength of my country's food management team is relatively weak. On this issue, the researchers said that the main factor that caused the problem was the relatively low professional ability and professionalism of the staff. Based on this, in the process of daily work, some staff often have difficulty applying

\footnotetext{
Copyright (C) 2021 Hailing Wang et al.

doi: 10.18686/fsr.v3i1.1368

This is an open-access article distributed under the terms of the Creative Commons Attribution Non-Commercial License

(http://creativecommons.org/licenses/by-nc/4.0/), which permits unrestricted non-commercial use, distribution, and reproduction in any medium, provided the original work is properly cited.
} 
relevant knowledge reasonably. The development and implementation of the work has had an adverse impact on the reasonable guarantee of food safety in my country and the effective detection of excessive additives in food. At the same time, by analyzing a large amount of data, the researchers pointed out that at this stage, some workers pay relatively little attention to food hygiene work. Among them, some people have not effectively realized the full understanding and importance of indoor hygiene work. Therefore, it is often difficult for them to reasonably realize the systematic analysis and exploration of related work in their daily work, resulting in the weakening of the comprehensive functions of the food management team in my country, which is extremely detrimental to the protection of food safety.

\subsection{There are still some loopholes in the food management system}

Through analyzing a large amount of data, the researchers pointed out that at this stage, the current food management system of my country's food hygiene department has certain deficiencies that need to be improved. Among them, in terms of details, the content of some systems is relatively vague and not effective. Realizing the elaboration of specific content is not conducive to the reasonable implementation of the staff's orderly implementation of specific tasks. At the same time, at this stage, my country's food hygiene work is jointly supervised by multiple departments. However, in this process, the rights and responsibilities of each department have not been reasonably analyzed and determined. Based on this, in the specific work, the rights and responsibilities between various departments are often difficult to achieve effective application, which leads to the problem of partial regional vacuum in supervision work, which is not conducive to the effective guarantee of food safety in my country, and is extremely detrimental to the reasonable maintenance of food safety for the broad masses of people.

\subsection{The level of technology used in food management is low}

In the process of food work, through analyzing a large amount of data, researchers pointed out that advanced food inspection technology often has an important guiding role in food safety work. However, looking at the current development of food work in my country, the food hygiene inspection technology used by some departments is relatively traditional. Based on this, in daily work, in the process of safety inspection of related foods, it is easy to cause errors in inspection results, which is not conducive to the guarantee of the accuracy of the results, and has an extremely adverse effect on the improvement of the overall quality of the inspection work. On the other hand, because the inspection technology is relatively backward, in the specific inspection work, facing the increasing number of inspected products, the inspection efficiency is often relatively low, which is not conducive to the timely detection and reasonable resolution of potential problems. The improvement of the basic efficiency of my country's food safety work has caused adverse effects and obstacles.

\subsection{Food management work supervision work needs to be improved}

At this stage, in the process of food management work, the food supervision and management departments in some regions have not effectively established and improved the supervision team. Based on this, in the process of daily work, the relevant departments have not reasonably realized the work of the supervisors. The systematic analysis of the situation is not conducive to the promotion and improvement of the comprehensive level of supervision work, and has an adverse effect on the improvement of the quality of supervision work in my country. On this issue, the researchers said that by combining advanced foreign food hygiene management experience, it can be found that a complete supervision system is conducive to timely detection and reasonable adjustment of potential safety hazards at work, and is good for improving the overall level of food work. Based on this, relevant departments should actively pay attention to and pay close attention to related issues, so as to formulate and improve response strategies based on their actual conditions, so as to provide a new impetus for the optimization of the overall level of my country's food work.

\section{Recommendations for optimizing the comprehensive level of food work}

2.1 Actively build a high-quality food man- 


\section{agement team}

From the staff's point of view, in order to further improve the overall level of food management in my country, the relevant departments should actively establish and improve the management team, so as to be effective for the relevant management team based on the actual situation of the staff. In terms of specific practices, the relevant departments can implement the implementation from two aspects. On the one hand, they should actively introduce advanced talents in a reasonable manner, so as to realize the full play of the role of talents and provide impetus for the improvement of the comprehensive quality of the internal work team of the department. On the other hand, for the existing management staff of the department, the relevant department should actively organize the study and discussion of relevant professional knowledge, so as to help the staff reasonably realize the understanding and mastery of advanced food hygiene knowledge, so as to help them in their daily work. The orderly development has laid a solid foundation and guarantee.

\subsection{Do a good job in perfecting the food management system according to the actual situation}

For the management department, in order to further realize the improvement and optimization of the food hygiene management level, the relevant departments should actively formulate and improve the management work system based on the actual situation, so as to guide the staff to further realize the reasonable development and optimization of the relevant work. Implementation, so as to provide help for the orderly progress of management. On this issue, the researchers pointed out that through the formulation and improvement of relevant systems, relevant departments can further realize the systematic clarification of their own rights and responsibilities, and have a good guiding role in the orderly practice of management. For example, by clarifying the inspection work system reasonably, it is helpful to help managers to further implement each step reasonably in the form of words, and it has a good promotion significance for the improvement of the quality of food inspection work.

\subsection{Effectively promote the reasonable in- troduction of advanced food safety inspec-}

\section{tion technology}

In general, in order to effectively improve the quality and efficiency of food safety inspection work, relevant departments should actively introduce and apply advanced inspection technology in their daily work, so as to continuously improve their own food inspection work level and efficiency, and provide food inspection. The improvement of work lays a solid foundation. On this issue, a large amount of data shows that through the application of advanced technology, food safety management personnel can better achieve reasonable detection of food samples, which is conducive to the realization of the content of harmful substances in the samples. Reasonable and clear information such as nutrient composition structure is vital to the improvement of the comprehensive level of my country's food safety inspection work.

\subsection{Deeply promote the effective establish- ment of food supervision work teams}

In daily work, in order to better implement the food management work and correct potential problems in a timely manner, the relevant departments should actively establish and improve the supervision team, so as to actively implement it according to the actual situation of my country's food work development at this stage. The revision and adjustment of the management system laid a solid foundation for the improvement of the overall quality of management work. At the same time, under the influence of the regulatory system, relevant staff can better realize their enthusiasm for work. It is conducive to further improving the efficiency of my country's food supervision. In addition, a large amount of data shows that through the reasonable implementation of the regulatory system, relevant departments can systematically analyze the development of food work, which has a good promotion significance for the solution of specific problems.

\section{Conclusion}

Researchers pointed out that, as one of my country's important social work, the development and implementation of food hygiene work is conducive to the promotion and optimization of the comprehensive level of food hygiene work in my country, and has a good promotion 
significance for the improvement of the comprehensive level of food work in my country. However, at present, there are still certain shortcomings in the development of my country's food work that need to be improved. Among them, the weakness of managers, the low level of management systems and technology, and the lack of regulatory systems all contribute to my country's management work. An important factor in the weakening of the level. On this issue, the researchers said that in order to reasonably achieve effective solutions to related issues, relevant departments should actively analyze related issues, and at the same time formulate and implement corresponding optimization suggestions and improvement strategies.

\section{References}

1. Zhang S, Yang Z. Experience and enlightenment of food safety risk management and liability insurance development in EU and Taiwan (in Chinese). Journal of Insurance Professional College 2015;
29(2): 74-76.

2. Qi H, Liu X. Thoughts and research on food safety and hygiene management in colleges and universities (in Chinese). China Market 2014; (25): 66-67.

3. Liang J, Guo R, Zeng S. The comparison study on management of health food safety and sanitation across the Taiwan Straits. Food and Nutrition in China 2014; 20(4): 10-14.

4. National Health and Family Planning Commission administrative measures for experts on food safety-related administrative license review (in Chinese). Chinese Journal of Health Inspection 2014; (2): 103-104.

5. Liu H. Investigation and analysis of the hygienic status of street snacks in a county and countermeasures (in Chinese). Journal of Medical Pest Control 2011; (2): 161, 163.

6. Zhang J, Wang Z. Sanitary status of bulk foods and its supervision measures in Lianyungang City. Occupation and Health 2011; 27(3): 295-296.

7. Yang Y. Discussion on the current situation and countermeasures of school food hygiene in Dali Prefecture, Yunnan Province (in Chinese). Chinese Primary Health Care 2009; 23(7): 59. 